\title{
Simulating Future GPS Clock Scenarios With Two Composite Clock Algorithms
}

\author{
Matthias Suess (DLR), Demetrios Matsakis (USNO) \\ and Charles A. Greenhall (JPL)
}

Matthias.suess@dlr.de

German Aerospace Centre (DLR)

Oberpfaffenhofen, Germany

\author{
Demetrios.Matsakis@usno.navy.mil \\ United States Naval Observatory (USNO), \\ Washington, D.C., USA \\ cgreenhall@jpl.nasa.gov \\ Jet Propulsion Laboratory (JPL), \\ Pasadena, CA, USA
}

\begin{abstract}
Using the GPS Toolkit, the GPS constellation is simulated using thirty-one satellites (SV) and a ground network of seventeen monitor stations (MS). At every 15 minute measurement epoch, the monitor stations measure the time signals of all satellites above a parameterized elevation angle. Once a day, the satellite clock estimates are uploaded to the satellites. Two composite clock algorithms are applied to estimate the station and satellite clocks. The first composite clock (B) is based on the Brown algorithm [1], and is now used by GPS. The second one $(G)$ is based on the Greenhall algorithm [2]. The composite clock of $G$ and $B$ performances are investigated using three ground clock models. Model C simulates the current GPS configuration, in which all stations are equipped with cesium clocks, except for masers at USNO and Alternate Master Clock (AMC) sites. Model M is an improved situation in which every station is equipped with active hydrogen masers. Finally, Model $F$ is a future scenario in which the USNO and AMC stations are equipped with fountain clocks instead of masers. Each model is evaluated using three performance metrics. The timing related user range error having all satellites available is the first performance index (PI1). The second performance index (PI2) relates to the stability of the broadcast GPS system time itself. The third performance index (PI3) evaluates the stability of the time scales computed by the two composite clocks.
\end{abstract}




\section{INTRODUCTION}

In the beginning, the GPS control segment consisted of 5 monitor stations including one master control station at Schriever, Air Force Base (AFB). Today, the data from 12 additional control stations operated by the National Geospatial-Intelligence Agency (NGA) were integrated to the GPS control segment (Figure 1) [1, 2]. Thus, a total of 17 monitor stations are available.

The monitor stations at Washington DC, USNO and Schriever, AFB are referenced to masers, while all other stations are equipped with HP 5071 Cesium clocks [3]. USNO is planning to replace the maser references in Washington and Schriever with rubidium fountains. The paper uses a simplified model of the operational GPS Kalman Filter, limited to clock estimates, to investigate by simulation the impact of improved monitor station clocks on the GPS timing performance.

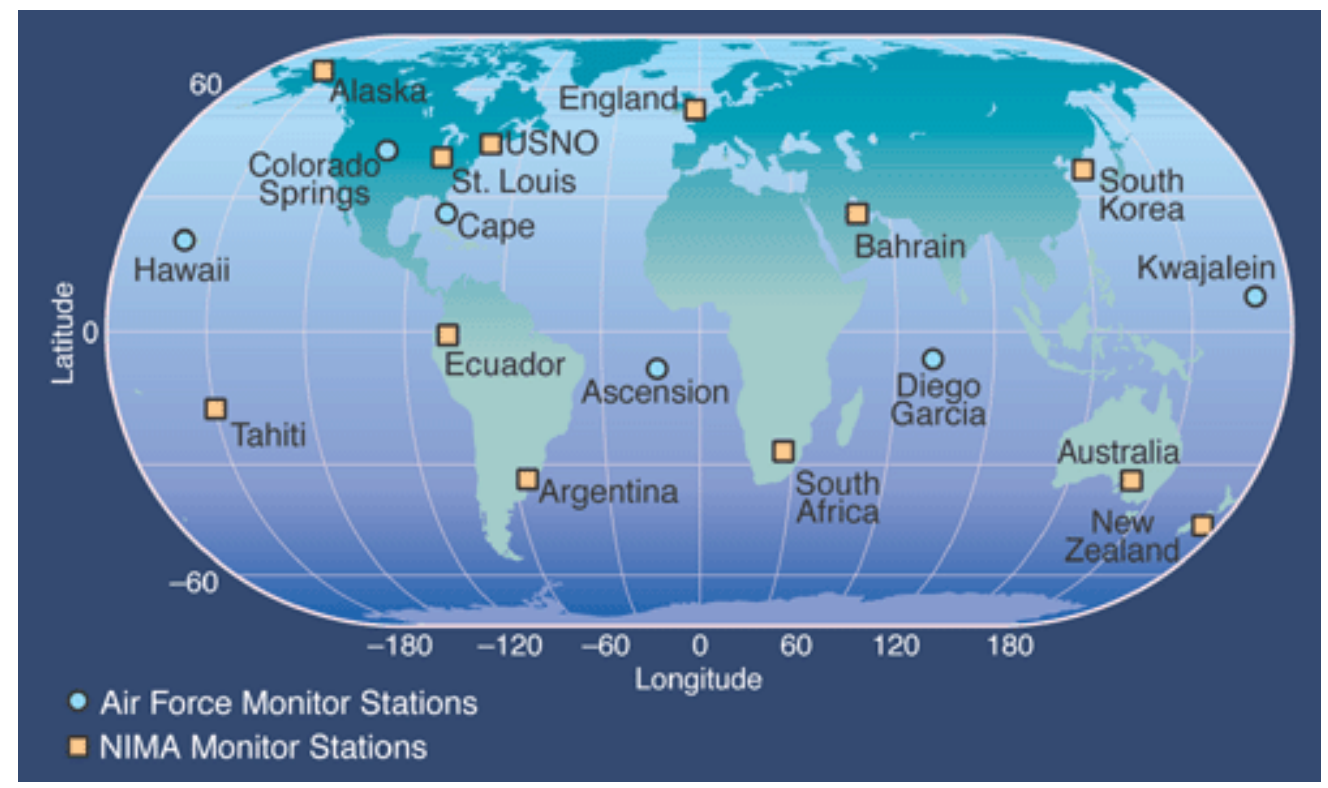

Figure 1: GPS ground segment

(http://www.aerospace.org/publications/crosslink/summer2002/04.html)

\section{SIMULATION OF THE GPS WORKFLOW}

Using the GPS toolkit software [4], the positions of 31 satellites are modeled based on the GPS almanac file of week 531. The satellites are equipped with Rubidium Atomic Frequency Standards (RAFS). Each monitor station observes the time offset, compared to its reference clock, of the satellites in view. The measurements are simulated with a 15-minute spacing, a white Gaussian noise of $0.7 \mathrm{~ns}$ ( 1 sigma), and an elevation angle mask of $20^{\circ}$.

The clock parameters computed by the Kalman Filter are uploaded to each satellite once a day as a broadcast model. In this simulation we assume the update occurs when the satellite is in view over Schriever and the clock broadcast parameters are older than one day. 
For this work, only clock parameters are solved for. In the operational filter, satellite orbit and other parameters are simultaneously included and the correlations among the full set of parameters would be expected to decrease the precision of the derived solutions. This would most likely decrease the differences we have computed between the clock models. However, double-differencing between satellite and station pairs allows one to solve for all other parameters without estimating clocks, and to solve for clocks in a second solution using the non-clock parameters as inputs.

\section{SIMULATION OF THREE IMPROVED MONITOR STATION CLOCKS AND SATELLITE CLOCKS}

Each clocks is modeled with a 3-state vector $\mathrm{X}(\mathrm{t})$, which is their phase, frequency, and drift offset $[5,6]$. It is modeled by the stochastic differential equation (SDE)

$$
\begin{gathered}
\frac{d X}{d t}(t)=\left(\begin{array}{lll}
0 & 1 & 0 \\
0 & 0 & 1 \\
0 & 0 & 0
\end{array}\right) X(t)+W(t) \\
E W(t) W^{T}(t)=\left(\begin{array}{ccc}
q_{1} & 0 & 0 \\
0 & q_{2} & 0 \\
0 & 0 & q_{3}
\end{array}\right)=Q(0 s)
\end{gathered}
$$

with a deterministic zero start value: $\mathrm{X}\left(\mathrm{t}_{0}\right)=0$.

The three q's measure the noise contributions from white frequency modulation (WFM), random walk frequency modulation (RWFM), and random run frequency modulation (RRFM). Neglecting some terms [6], the q's can be related to the Allan and Hadamard Deviations as:

$$
\begin{gathered}
A D E V^{2}(\tau)=q_{1} \tau^{-1}+\frac{1}{3} q_{2} \tau+\frac{1}{20} q_{3} \tau^{3}+\ldots \\
\operatorname{HDEV}^{2}(\tau)=q_{1} \tau^{-1}+\frac{1}{6} q_{2} \tau+\frac{11}{120} q_{3} \tau^{3}+\ldots
\end{gathered}
$$

The discrete solution of the SDE, with $\tau$ equal to $15 \mathrm{~min}$, is used to simulate the clock types $[6,7,8]$. Following the assumptions of the Kalman Filter, it can be shown that the process noise matrix $Q(\tau)$ is a simple function of the q's and the filter time spacing $\tau$ :

$$
Q(\tau)=\left(\begin{array}{ccc}
q_{1} \tau+q_{2} \frac{1}{3} \tau^{3}+q_{3} \frac{1}{20} \tau^{5} & q_{2} \frac{1}{2} \tau^{2}+q_{3} \frac{1}{8} \tau^{4} & q_{3} \frac{1}{6} \tau^{3} \\
q_{2} \frac{1}{2} \tau^{2}+q_{3} \frac{1}{8} \tau^{4} & q_{2} \tau+q_{3} \frac{1}{3} \tau^{3} & q_{3} \frac{1}{2} \tau^{2} \\
q_{3} \frac{1}{6} \tau^{3} & q_{3} \frac{1}{2} \tau^{2} & q_{3} \tau
\end{array}\right)
$$

Three different types of monitor station clocks are simulated: cesium, active hydrogen maser (AHM) and fountain clocks. Their stochastic components (q's) together with the RAFS are 
specified in table 1, and typical Allan deviations are shown in Figure 2. The values for these q's should not be taken as authoritative by any means, nor should the associated Allan deviations be assumed as anything but a rough approximation.

\begin{tabular}{|c|c|c|c|}
\hline & $\mathbf{q}_{\mathbf{1}} \mathbf{W F M}\left[\mathbf{s}^{2} / \mathbf{s}\right]$ & $\mathbf{q}_{2}$ RWFM $\left[\mathbf{s}^{2} / \mathbf{s}^{3}\right]$ & $\mathbf{q}_{\mathbf{3}}$ RRFM $\left[\mathbf{s}^{2} / \mathbf{s}^{\mathbf{5}}\right]$ \\
\hline Cesium & $2.50 \mathrm{e}-23$ & $4.44 \mathrm{e}-37$ & $5 \mathrm{e}-53$ \\
\hline AHM & $2.8 \mathrm{e}-26$ & $1.1 \mathrm{e}-35$ & $4.4 \mathrm{e}-51$ \\
\hline Fountain & $4.4 \mathrm{e}-27$ & $1.1 \mathrm{e}-37$ & $1.1 \mathrm{e}-55$ \\
\hline RAFS & $1.0 \mathrm{e}-24$ & $1.1 \mathrm{e}-35$ & $2.8 \mathrm{e}-46$ \\
\hline
\end{tabular}

Table 1: Clock noise models.

Since the data are assumed to be processed in 15-minute batches, the printed values are scaled by factor of 900 from the rounded-off values used to generate the time series.

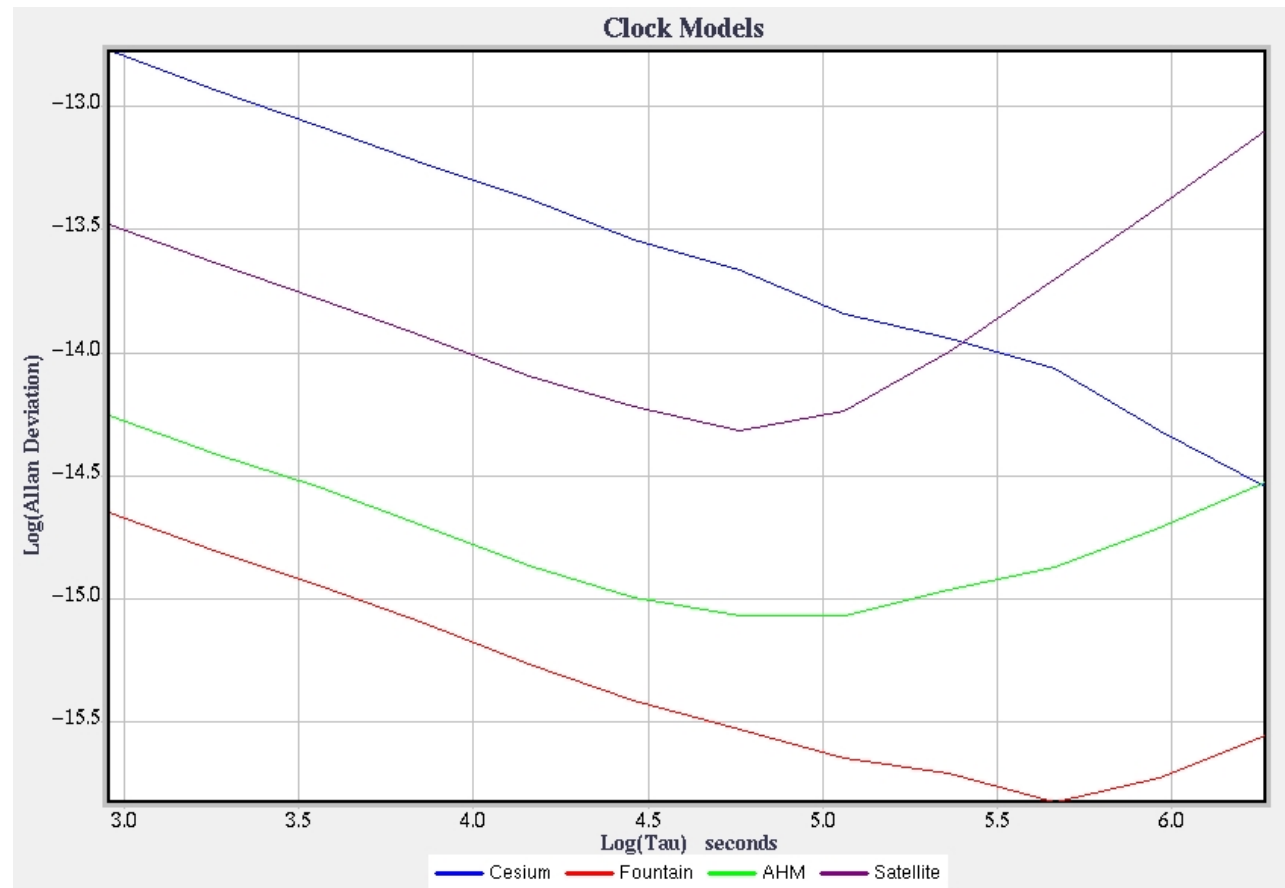

Figure 2: Allan deviation of one individual clock simulation of each modeled type. (It assumed that the necessary resources are expended to keep the masers and the atomic fountains within their environmental specifications.)

For numerical issues it is found to be useful to change the units of the q values (table 1) which are conventionally given in seconds by seconds to nanoseconds by day:

$$
\left(\frac{10^{18}}{86400^{-1}} q_{1} \frac{10^{18}}{86400^{-3}} q_{2} \quad \frac{10^{18}}{86400^{-5}} q_{3}\right)\left[\begin{array}{lll}
\frac{n s^{2}}{d} & \frac{n s^{2}}{d^{3}} & \frac{n s^{2}}{d^{5}}
\end{array}\right]
$$

\section{COMPOSITE CLOCK ALGORITHMS - BROWN AND GREENHALL}

Two composite clock algorithms [7,8] are applied to process the time offset measurements between the control stations and the satellite clocks and to estimate the satellite and control station clocks with respect to its implicitly defined time scales. Since N clocks involve 
maximal N-1 linearly independent measurements, each composite clock algorithm includes a method to prevent the formal covariance of the solution parameters from growing without bound. The covariance reductions further obscure intuitive understanding of the solutions, but protects against computer limitations in handling large numbers.

\section{THE BROWN COMPOSITE CLOCK}

It can be shown [7] that no measured quantity is affected if the Kalman Filter covariance estimates $\mathrm{C}(\mathrm{t})$ are reduced at each iteration step as follows:

$$
B(C(t))=C(t)-\bar{H}\left(\bar{H}^{T} C^{-1}(t) \bar{H}\right)^{-1} \bar{H}^{T}
$$

with

$$
\bar{H}=\left(\begin{array}{c}
I_{3} \\
\vdots \\
I_{3}
\end{array}\right) \in R^{3 N \times 3}
$$

Sorting the covariance by the states more clearly illustrates the operation of the Brown reduction. Each $\mathrm{NxN}$ sub matrix $\mathrm{C}_{\mathrm{ij}}$ with $i, j \in\{x, y, d\}$ is subtracted by a $\mathrm{NxN}$ matrix with a common element defined by $\mathrm{c}_{\mathrm{ij}}$

$$
C=\left(\begin{array}{lll}
C_{x x} & C_{x y} & C_{x d} \\
C_{y x} & C_{y y} & C_{y d} \\
C_{d x} & C_{d y} & C_{d d}
\end{array}\right) \rightarrow B(C)=\left(\begin{array}{ccc}
C_{x x} & C_{x y} & C_{x d} \\
C_{y x} & C_{y y} & C_{y d} \\
C_{d x} & C_{d y} & C_{d d}
\end{array}\right)-\left(\begin{array}{lll}
1_{N, N} C_{x x} & 1_{N, N} C_{x y} & 1_{N, N} C_{x d} \\
1_{N, N} C_{y x} & 1_{N, N} C_{y y} & 1_{N, N} C_{y d} \\
1_{N, N} C_{d x} & 1_{N, N} C_{d y} & 1_{N, N} C_{d d}
\end{array}\right)
$$

with

$$
\left(\bar{H}^{T} C^{-1}(t) \bar{H}\right)^{-1}=\left(\begin{array}{ccc}
c_{x x} & c_{x y} & c_{x d} \\
c_{y x} & c_{y y} & c_{y d} \\
c_{d x} & c_{d y} & c_{d d}
\end{array}\right) \text { and } 1_{N, N}=\left(\begin{array}{ccc}
1 & \cdots & 1 \\
\vdots & & \vdots \\
1 & \cdots & 1
\end{array}\right) \in R^{N x N}
$$

As a test of the programming, we have computed solutions with and without the covariance reduction, and verified that all quantities seen by the user are indeed unchanged. The Kalman filter is executed every 15 minutes and, after every iteration of the Kalman filter the Brown reduction is applied. It results a series of reduced covariances which are called:

$$
C_{B}\left(t_{k}\right)=B\left(F\left(C_{B}\left(t_{k-1}\right)\right)\right)
$$

with the matrix function $\mathrm{F}$ models the Kalman filter iteration. The initial values are

$$
\hat{X}\left(t_{0}\right)=0 \text { and } C_{B}\left(t_{0}\right)=1 e^{10} Q(\tau)
$$


The reduction method by Greenhall is of a similar manner; however it works on the sub matrix $\mathrm{C}_{\mathrm{xx}}$ of the phase states [8]. Using the auxiliary matrix

$$
S=\left(\begin{array}{ccc}
A & 0 & 0 \\
0 & I_{N} & 0 \\
0 & 0 & I_{N}
\end{array}\right)
$$

with

$$
A=\left(I_{N}-1_{N} 1_{N}^{T}\left(1_{N}^{T} C_{x x}^{-1} 1_{N}\right)^{-1}\right) \text { and } 1_{N}=\left(\begin{array}{c}
1 \\
\vdots \\
1
\end{array}\right) \in R^{N}
$$

the Greenhall reduction is defined by

$$
G(C(t))=S C(t) S^{T}
$$

The matrix operation only affects the sub-matrixes involving the phase-parts:

$$
C=\left(\begin{array}{ccc}
C_{x x} & C_{x y} & C_{x d} \\
C_{y x} & * & * \\
C_{d x} & * & *
\end{array}\right) \rightarrow G(C)=\left(\begin{array}{ccc}
C_{x x}^{*} & A C_{x y} & A C_{x d} \\
C_{x y} A^{T} & * & * \\
C_{x d} A^{T} & * & *
\end{array}\right)
$$

with

$$
C_{x x}^{*}=C_{x x}-1_{N}\left(1_{N}^{T} C_{x x}^{-1} 1_{N}\right)^{-1} 1_{N}^{T}
$$

As pointed out by Greenhall [8], running the Kalman filter with his reduction method changes the phase estimates but not the frequency and drift estimates. The reduced covariance of the Greenhall composite clock is defined by:

$$
G\left(F\left(C_{G}\left(t_{k-1}\right)\right)\right)=C_{G}\left(t_{k}\right)
$$

The same initial values as in case of Brown are used.

Using the Kalman filter with the Brown reduction is called the Brown composite clock, whereas running the Kalman filter with the Greenhall reduction is called the Greenhall composite clock. 


\section{THREE GROUND SEGMENT MODELS}

Table 2 outlines three different ground segment models for the stability of the monitor station clocks.

\begin{tabular}{|c|l|}
\hline Model C & $\begin{array}{l}\text { Cesium at every station except for AHMs at } \\
\text { Schriever and USNO }\end{array}$ \\
\hline Model M & AHMs at every station \\
\hline Model F & $\begin{array}{l}\text { Cesiums at every station except at Schriever } \\
\text { and USNO, where USNO will maintain } \\
\text { rubidium-based atomic fountains }\end{array}$ \\
\hline
\end{tabular}

Table 2: model definition

Model C describes the current situation in GPS whereas Models M and F are possible future scenarios.

\section{PERFORMANCE INDICES 1, 2 AND 3}

In order to assess the different models three performance indices (PI) are defined. The following nomenclature is used

$$
X=\left(\begin{array}{l}
x \\
y \\
d
\end{array}\right) \text { and } \hat{X}=\left(\begin{array}{c}
\hat{x} \\
\hat{y} \\
\hat{d}
\end{array}\right)
$$

with $x, y, d \in R^{N}$ the phase, frequency and drift simulations and $\hat{x}, \hat{y}, \hat{d} \in R^{N}$ the corresponding estimates.

\section{PI 1: THE TIME RELATED USER RANGE ERROR}

The first index measures the accuracy of the clock broadcast models (BM), which are the latest uploads of their three-state Kalman filter estimates. For each satellite k, the broadcast model is updated at least every 24 hours and consists of three parameters used by the users to correct the clock:

$$
B M_{k}(t)=a_{0}+a_{1}\left(t-t_{\text {upload }}\right)+\frac{1}{2} a_{2}\left(t-t_{\text {upload }}\right)^{2}
$$

For each phase value $x_{k}(t)$ of clock $\mathrm{k}$ at time $\mathrm{t}$, the phase estimation error in this correction is given by

$$
\tilde{x}_{k}(t)=x_{k}(t)-B M_{k}(t)
$$

The empirical standard deviation of the corrected satellite clocks $\mathrm{k}$ using the available time samples i (excluding the first day data) defines the PI1: 


$$
P I 1=\sigma_{x}\left(\left\{\tilde{x}_{k}\left(t_{i}\right) \mid k, i\right\}\right)
$$

PI1 is understandable as the average asynchronization of the broadcast model-corrected clocks at time $t$. It is a measure of the signal-in-space limitations to real-time positioning or synchronization with GPS. In order to allow the clock parameter determinations to mature (become independent of initial assumptions), PI1 (and PI2), are computed excluding the first day's data.

\section{PI2: THE STABILITY OF THE BROADCASTED GPS SATELLITE TIME}

The second performance index, PI2, evaluates the stability of the average GPS satellite time as broadcast:

$$
P I 2(\tau)=A D E V\left(\frac{1}{31} \sum_{k=1}^{31} \tilde{x}_{k}(t), \tau\right)
$$

\section{PI 3: THE TIME SCALE STABILITY}

Both composite clocks define different time scales within the Kalman filter. In case of the Greenhall composite clock, the time scale weights are given by

with

$$
w_{G}(t)=C_{G, X x}^{-1}(t) 1_{N}\left(1_{N}^{T} C_{G, x x}^{-1}(t) 1_{N}\right)^{-1}
$$

$$
C_{G}(t)=\left(\begin{array}{lll}
C_{G, x x}(t) & C_{G, x y}(t) & C_{G, x d}(t) \\
C_{G, y x}(t) & C_{G, y y}(t) & C_{G, y d}(t) \\
C_{G, d x}(t) & C_{G, d y}(t) & C_{G, d d}(t)
\end{array}\right)
$$

and its time scale is

$$
\operatorname{sys}_{G}(t)=w_{G}^{T}(t) \tilde{x}(t)
$$

In contrast, the time scale of the Brown composite clock is the phase of the implicit ensemble mean. Using the Brown weight of all states

$$
\left.w_{B}^{T}(t)=\bar{H}^{T} C_{B}^{-1}(t) \bar{H}\right)^{-1} \bar{H}^{T} C_{B}^{-1}(t)(1,:)
$$

it is defined by

$$
\operatorname{sys}_{B}(t)=w_{B}^{T}(t)\left(\begin{array}{c}
\tilde{x}(t) \\
\tilde{y}(t) \\
\tilde{d}(t)
\end{array}\right)
$$

The Brown time scale includes the corrected frequency and drift estimates as well. The third performance index, PI3, assesses the stability of GPS Time as computed within the two composite clocks. 


$$
\operatorname{PI3}\left(\tau, \text { sys. }_{\bullet}(t)\right)=A D E V\left(\text { sys. }_{\bullet}(t), \tau\right)
$$

Assuming the time $t$ is large enough for the parameters to fully mature, PI3 is stationary and given by the Allan Deviation of the system time.

For comparison reason the tau-weighted ADEV is defined as:

$$
A D E V_{\text {tau-weighted }}^{2}(\tau)=\sum_{k=1}^{N} w_{k}^{2}(\tau) A D E V_{k}^{2}(\tau)
$$

with weights

$$
w_{k}(\tau)=\left(\sum_{i=1}^{N} \frac{1}{A D E V_{i}^{2}(\tau)}\right)^{-1} \frac{1}{A D E V_{k}^{2}(\tau)}
$$

and $A D E V_{k}(\tau)$ theoretical Allan deviation of clock $\mathrm{k}[9]$.

\section{EVALUATION OF THREE MODELS}

\section{PERFORMANCE INDEX 1 AND 2}

Figure 3 show very little difference between models C, F, and M in PI1, which is a measure of GPS's real-time signal-in-space performance. This is expected because any improvements in the predictive ability should be masked by the individual satellite clocks' stochastic variations over the 24 hours between uploads. The Greenhall composite clock lightly outperforms Brown's for all models.

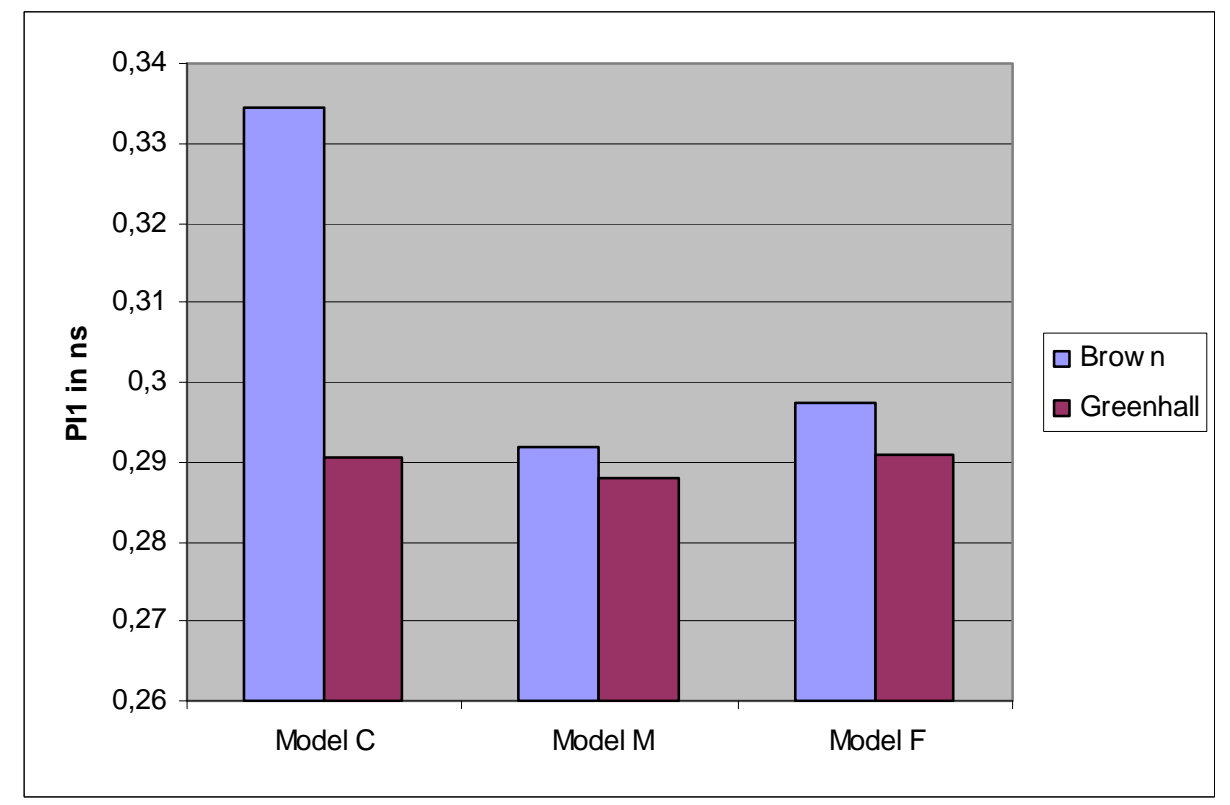

Figure 3: comparison of the PI1 for the three scenarios and the two composite clocks 
Similar results are shown in Figure 4 and 5, which is how PI2 would be computed by a worldwide array of receivers that did not have direct access to the GPS ground clocks and could only observe satellite broadcasts.

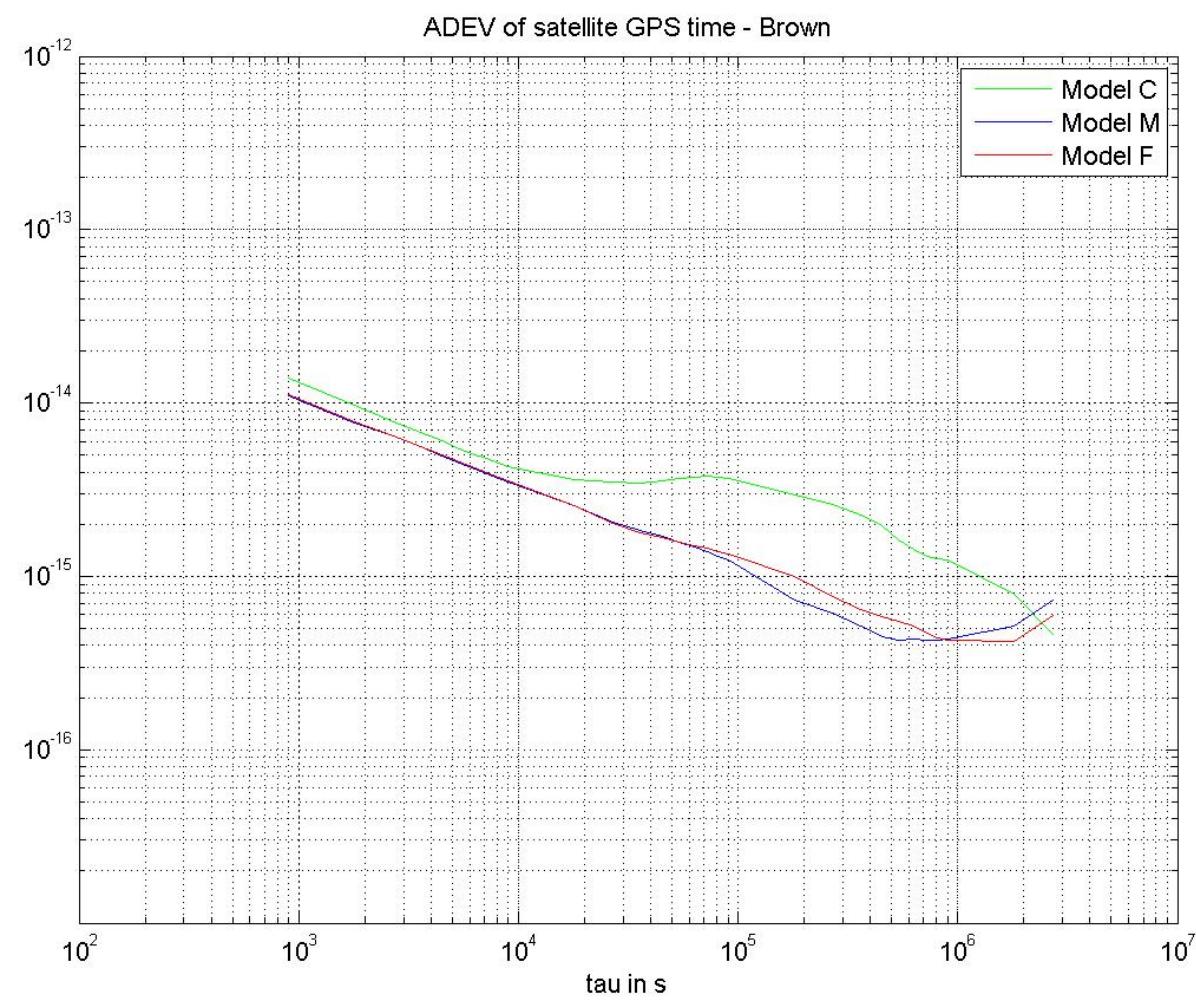

Figure 4: PI2 using Brown composite clock

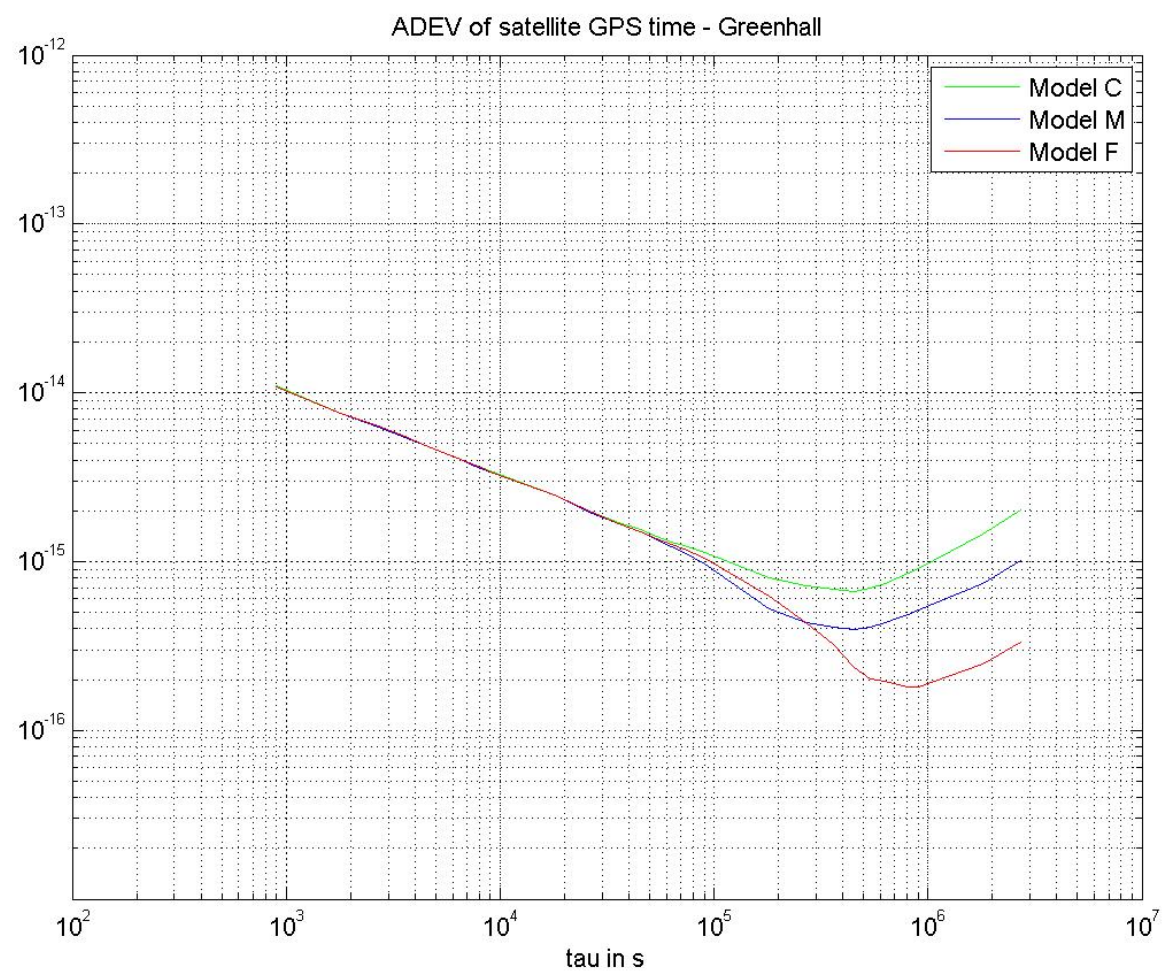

Figure 5: PI2 using Greenhall composite clock 


\section{PERFORMANCE INDEX 3}

Figure 6 compares the Allan deviations of the Brown and Greenhall time scales for each model. For both composite clocks, the stability improves for Model M and F compared to Model C.

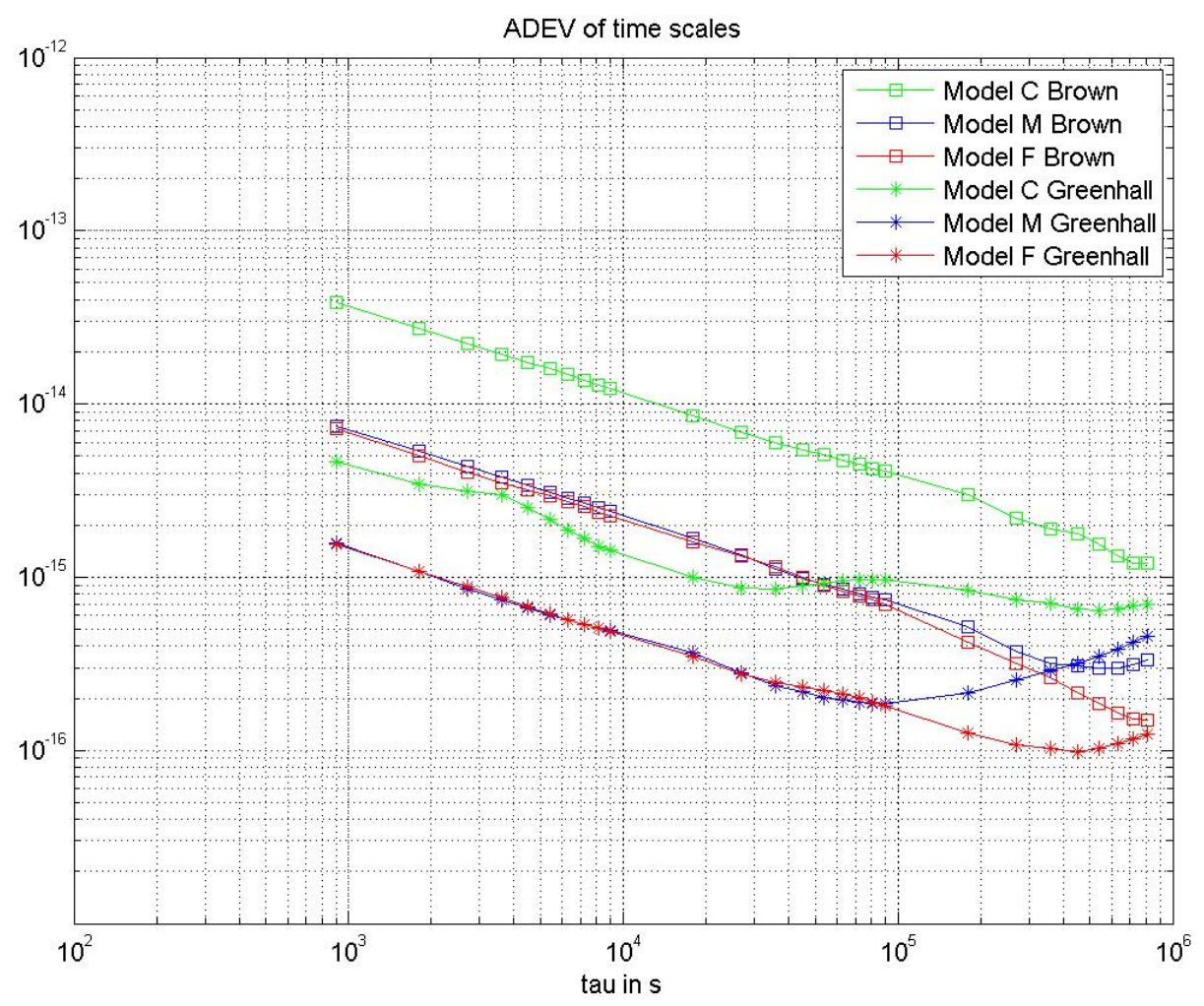

Figure 6: PI3 of the three models and the two composite clocks

The Greenhall time scale is more stable than the Brown time scale for any model. Since the model parameters for atomic fountains result in one fountain being roughly as stable as the RSS stability of an average of five masers for averaging times smaller than one day, it is also not surprising that in an 17-station solution Models $\mathrm{M}$ and $\mathrm{F}$ are roughly equivalent and also more stable than Model C.

The PI3 values given in Figure 6 show that use of better ground clocks (model M and F) would result in GPS Time being more stable with regards to UTC. We note that actual GPS Time is stabilized through steering to UTC(USNO). This steering becomes significant on the scale of a few days, and is ignored here. It also follows that the stability of GPS Time itself is only relevant to the extent it minimizes the amount of steering applied to GPS Time. Even though GPS Time is intended for navigational purposes only and users interested in UTC should apply the corrections broadcast in subframe 4 page 18 of the GPS Navigation message to derive UTC(USNO) directly, we can assume that the stability of GPS Time will translate into the stability of GPS's delivered prediction of UTC(USNO).

Another and closely related effect of improved PI3 is to decrease the required steering of GPS Time and the uncertainty in the broadcast corrections for UTC(USNO). 
Figure 7, 8 and 9 compares the two time scales, the tau-weighted ADEV and the participating clock ADEVs for each model. In case of model C (figure 7), the Greenhall time scale and the tau-weighted ADEV are close to each other for averaging times smaller than 1 day and more stable than the most stable clock of the ensemble, the AHM. The Brown stability (squares) violates this condition and most likely follows the average of the Cesium clocks (solid).

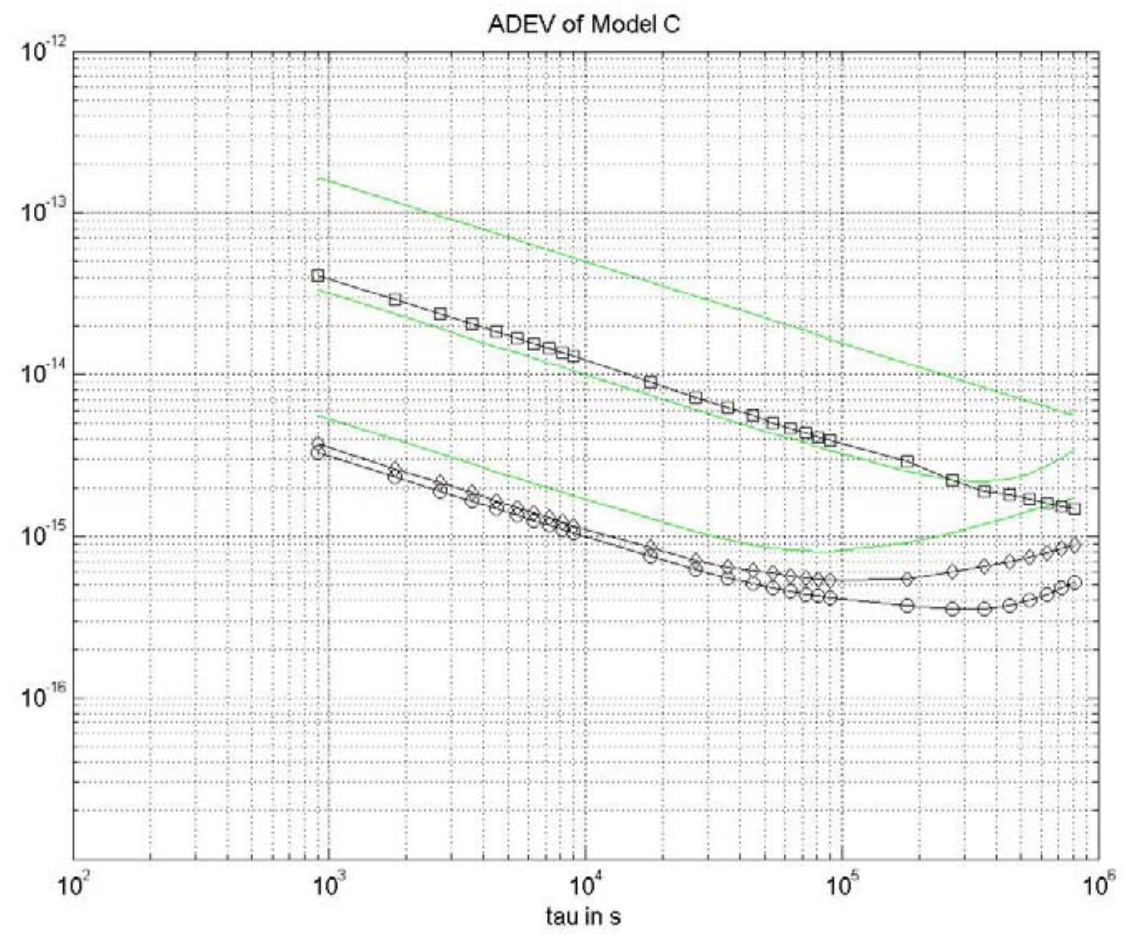

Figure 7: PI3 of the three models and the two composite clocks

Square: Brown time scale, Diamond: Greenhall time scale, Circle: tau-weighted ADEV, Solid: Cesium, RAFS, AHM

In case of model $\mathrm{M}$, the tau-weighted $\mathrm{ADEV}$ and the Greenhall time scale are close to each other and, again, more stable than the most stable ensemble clock (AHM). The Brown time scale is less stable than the AHM for averaging times shorter than one day. It looks like that the Brown time scale follows the average stability of the RAFS. 


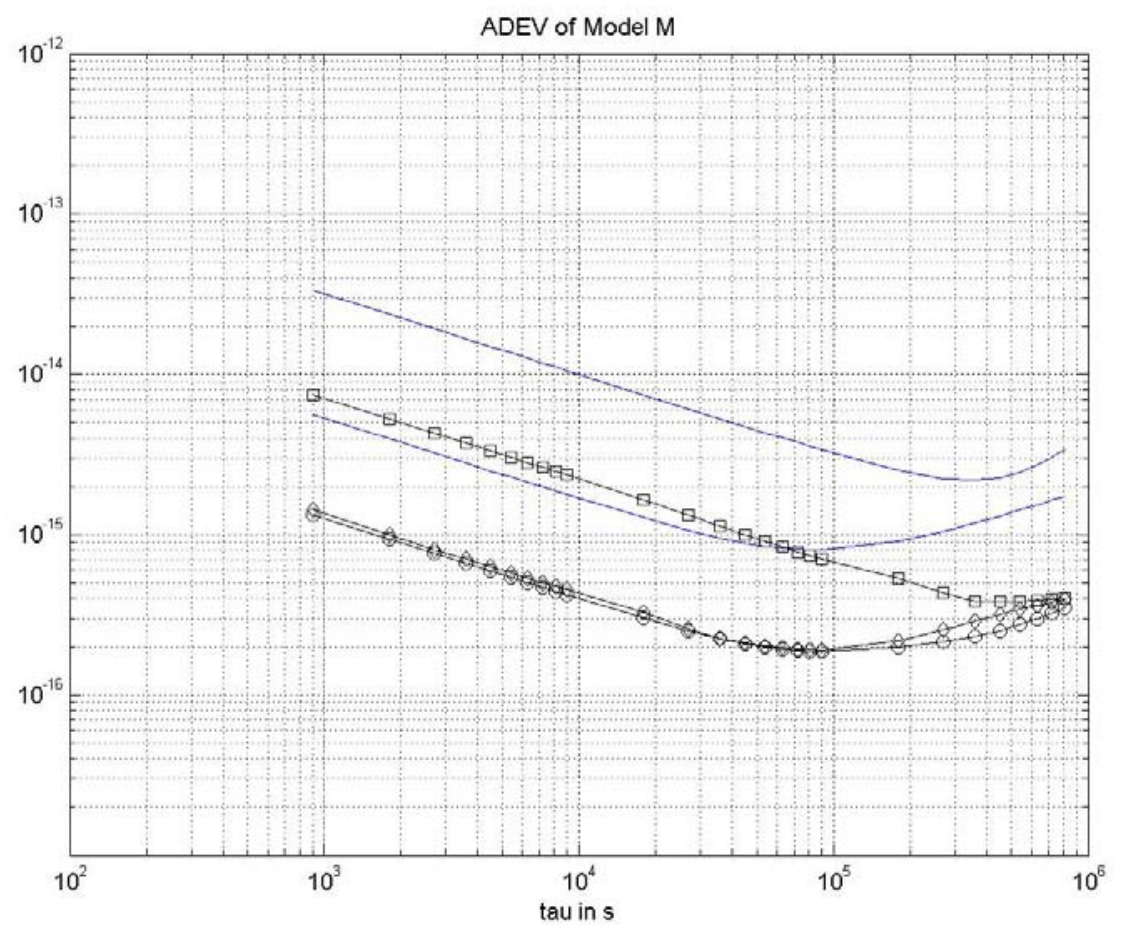

Figure 8: PI3 of the three models and the two composite clocks Square: Brown time scale, Diamond: Greenhall time scale, Circle: tau-weighted ADEV, Solid: RAFS, AHM

In case of Model F, the tau-weighted ADEV and the Greenhall times scale are close to each other and are more stable than the most stable clock (Fountain) for every averaging time. The Brown time scale violates this condition. 


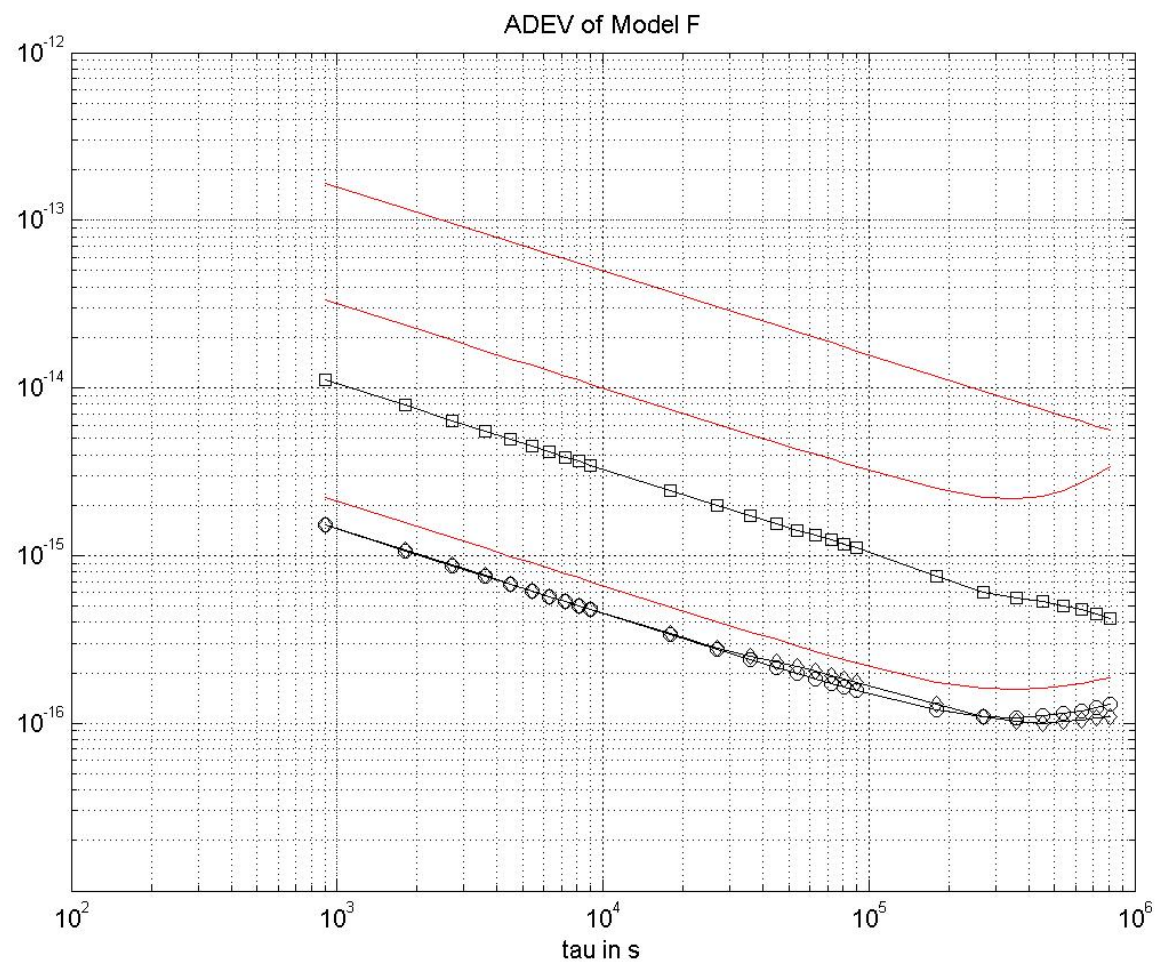

Figure 9: PI3 of the three models and the two composite clocks

Square: Brown time scale, Diamond: Greenhall time scale, Circle: tau-weighted ADEV, Solid: Cesium, RAFS, Fountain

\section{IMPACT OF MEASUREMENT NOISE AND MISS-TUNING OF $Q$ VALUES}

Figure 10 shows the impact of ten times more and less measurement noise on the performance index 1. Since the impact is similar for all models, model $\mathrm{F}$ is chosen exemplary. The figure shows the quotient between the nominal PI1 and the test PI1. Obviously, more measurement noise decreases the performance around 50\% whereas less measurement noise barely helps. The impact is similar for both composite clocks.

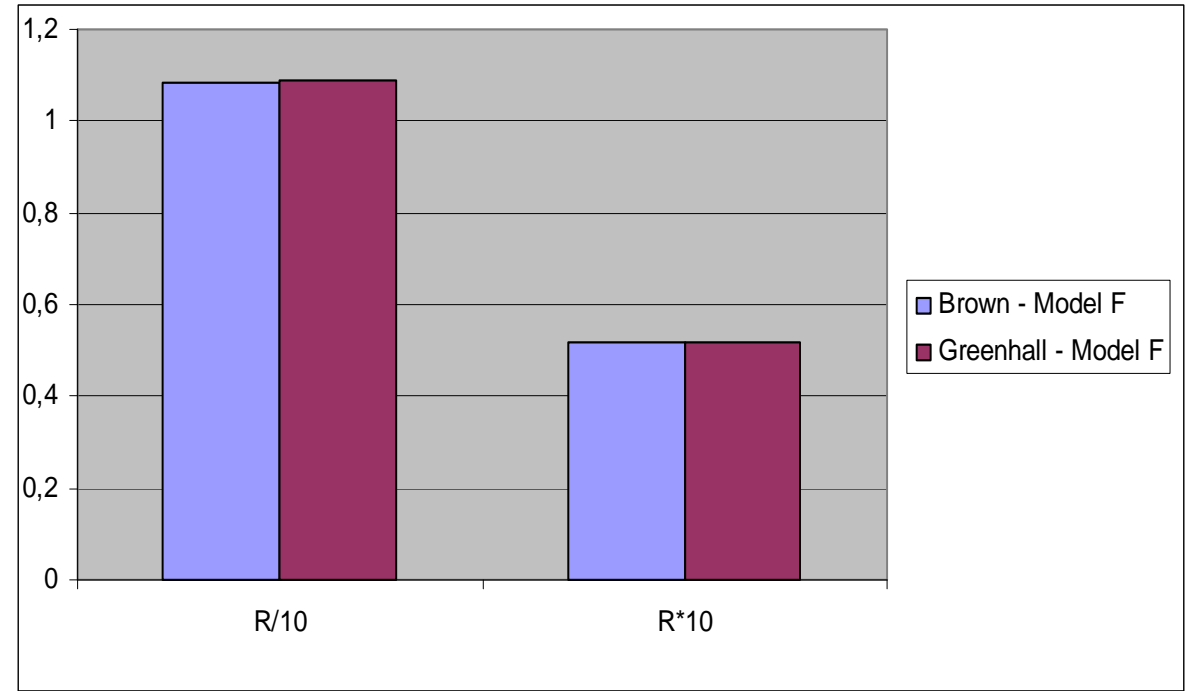


Figure 10: Impact of R variations on PI1 of model F

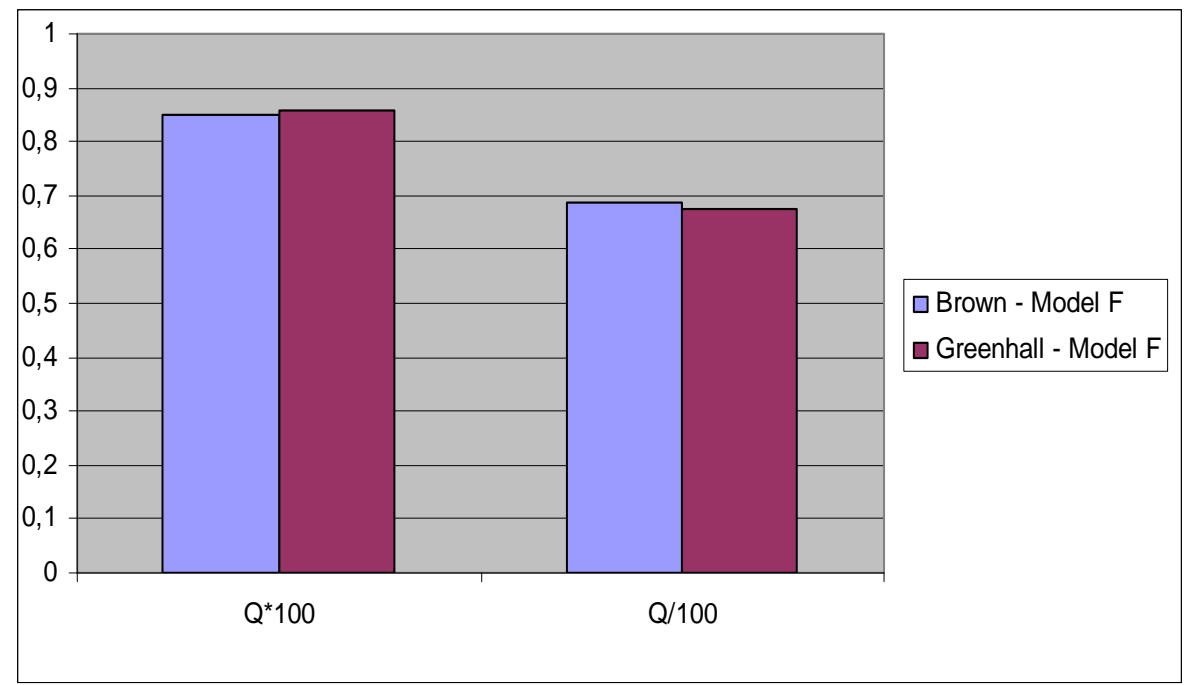

Figure 11: Impact of Q miss-modeling on PI1 on model F

Figure 11 shows the impact of miss-modeling the Q values on PI1. Two cases are simulated: the first one applies a 100 times higher and the second a 100 times smaller $Q$ value. We note that the clock simulations are not changed and are based on the right $\mathrm{Q}$. The quotient of the nominal PI1 and the test PI1 decreases in both cases, thus, the performance decreases. This is an indication that the Kalman filter is tuned right. This is the case for both composite clocks.

\section{COMBINATION OF GREENHALL AND BROWN REDUCTION}

Both reduction methods generate a time series of covariance matrices and estimates. An empirical study is performed which investigates the impact on the estimates and covariances of combining both methods. The two combinations are:

$$
B\left(G\left(F\left(C_{B(G)}\left(t_{k-1}\right)\right)\right)\right)=C_{B(G)}\left(t_{k}\right) \text { and } G\left(B\left(F\left(C_{G(B)}\left(t_{k-1}\right)\right)\right)\right)=C_{G(B)}\left(t_{k}\right)
$$

The simulations point out that both combinations compute the same estimates as just using the Greenhall reduction. Furthermore, the operations commute and compute the same covariance

$$
C_{B(G)}\left(t_{k}\right)=C_{G(B)}\left(t_{k}\right)
$$

The estimates are the same of the Greenhall composite clock:

$$
\hat{X}_{B(G)}\left(t_{k}\right)=\hat{X}_{G(B)}\left(t_{k}\right)=\hat{X}_{G}\left(t_{k}\right)
$$

The benefit of the combination is that also the frequency and drift parts of the Greenhall covariance are reduced by the Brown reduction. This is illustrated by figure 12. The upper plot shows the 


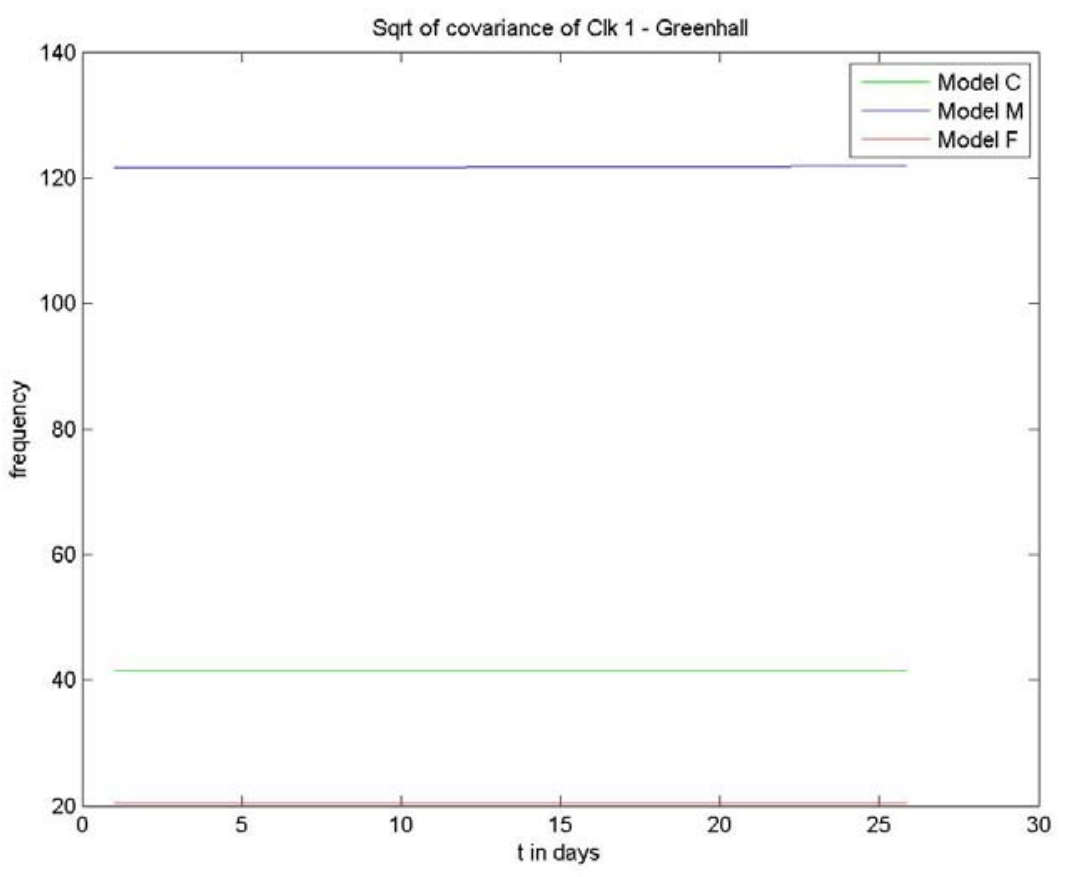

Figure 12: Greenhall frequency covariance of clock 1

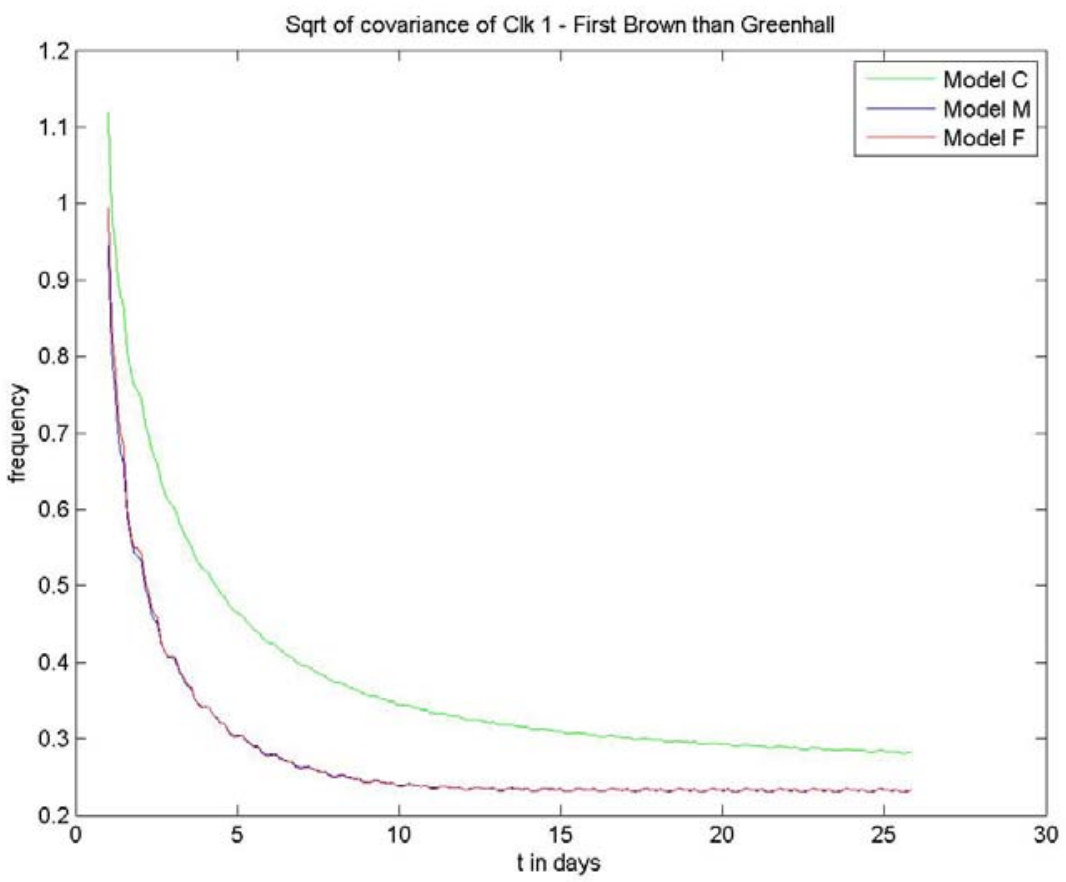

Figure 13: First Brown than Greenhall frequency covariance of clock 1

frequency covariance of clock 1 using the Greenhall reduction. Obviously, the frequency covariance is not reduced. Figure 13 shows the effect of combining Brown and Greenhall. Clearly, the covariance is reduced. 


\section{CONCLUSIONS}

Using a limited set of ground stations and a highly simplified model for the operational GPS Kalman Filter, it has been shown that the real-time positioning ability of GPS is insensitive to the improved ground clocks and composite clocks.

In contrast, GPS Time within the Kalman Filter would be more stable if all GPS sites had environmentally-protected masers or the USNO-controlled sites had atomic fountains. Its stability can be additionally increased applying the Greenhall composite clock. The Greenhall composite clock shows the most promising results for each model.

Furthermore, a combination of both reduction methods is suggested to instead of running the Greenhall reduction alone. The clock estimates are unchanged, and the frequency and drift parts of the covariance are reduced. The improvement by applying both covariance reduction algorithms are reported as empirical here, but one of us (CAG) intends to publish a mathematical proof in the near future.

\section{ACKNOWLEDGEMENTS}

We thank Benjamin Harris and the University of Texas for use of the GPS Toolkit.

\section{REFERENCES}

[1] http://www.aero.org/education/primers/gps/elements.html

[2] http://www.kowoma.de/en/gps/control_segment.htm

[3] D. Manning, "AF/NGA GPS Monitor Station High-Performance Cesium Frequency Standard Stability 2007/2008: From NGA Kalman Filter Clock Estimates”, 40th Precise Time and Time Interval Meeting (PTTI), 2008

[4] Timothy J. H. Craddock, Richard John Broderick, Colin P. Petersen, and Alex Hu "The GPS Toolkit: Open Source Clock Tools" 40th Precise Time and Time Interval Meeting (PTTI), 2008

[5] Zucca C. and Tavella P. "The clock model and its relationship with the Allan and related variances” IEEE Transactions on Ultrasonics, Ferroelectris and Frequency Control, 2005, 52

[6] Chaffee J. W. "Relating the Allan Variance to the Diffusion Coefficients of a Linear Stochastic Differential Equation Model for Precision Oscillators", IEEE Transactions on Ultrasonics, Ferroeletrics and Frequeny Control, 1987, UFFC-34

[7] Brown K. R., "The theory of the GPS composite clock”, Proc. ION GPS-91 Albuquerque NM, 1991

[8] Greenhall C. A., "A Kalman filter clock ensemble algorithm that admits measurement noise”, Metrologia 43 S311-S321

[9] Davis J. A., C. A. Greenhall, P. W. Stacey, "A Kalman filter clock algorithm for use in the presence of flicker frequency modulation noise”, Metrologia 42 (2005) 